\title{
CHIANTI - an atomic database for emission lines
}

\author{
III. Continuum radiation and extension of the ion database

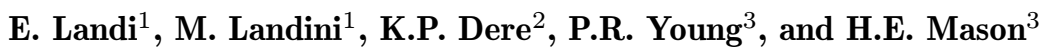 \\ 1 Department of Astronomy and Space Science, University of Florence, Italy \\ 2 Naval Research Laboratory, Washington DC, U.S.A. \\ 3 Department of Applied Mathematics and Theoretical Physics, University of Cambridge, UK
}

Received August 10; accepted October 9, 1998

\begin{abstract}
CHIANTI provides a database of atomic energy levels, wavelengths, radiative transition probabilities and electron excitation data for a large number of ions of astrophysical interest. This database allows the calculation of theoretical line emissivities necessary for the analysis of optically thin emission line spectra, and includes also a suite of IDL (Interactive Data Language) programs to calculate optically thin synthetic spectra and to perform spectral analysis and plasma diagnostics.

The first version (1.0) of the CHIANTI database was released in 1996 (Dere et al. 1997) and has been extensively used for analysis of line spectra from astrophysical sources by the scientific community.

The present paper describes the first major update to the CHIANTI database which will subsequently be labeled version 2.0. The update consists of the addition of a large number of new ions, the revision of existing data for some ions and the inclusion of an IDL procedure to calculate the continuum.

The CHIANTI atomic database and supporting IDL routines are freely available through the internet.

Key words: atomic data - astronomical data bases: miscellaneous - ultraviolet: general — Sun: atmosphere - stars: atmosphere - atomic database
\end{abstract}

\section{Introduction}

The main goal of CHIANTI is to provide the scientific community with a database that includes the best available calculations of atomic parameters for all ions of astrophysical interest and a suite of IDL routines for the analysis of astrophysical emission line spectra. The first

Send offprint requests to: E. Landi (enricol@arcetri.astro.it) version of the CHIANTI database has been released in 1996 (Dere et al. 1997, hereafter Paper I) and concerned the optically thin emission line spectrum for wavelengths greater than $50 \AA$.

Since then, several new theoretical calculations of atomic parameters have become available for a variety of new ions, which represent great improvements from the previous data. Moreover, the Coronal Diagnostic Spectrometer (CDS - Harrison et al. 1995) and Solar Ultraviolet Measurements of Emitted Radiation (SUMER - Wilhelm et al. 1995) instruments on board of the Solar and Heliospheric Observatory (SOHO) have recorded many spectra between $170 \AA$ and $1600 \AA$ (e.g., Harrison et al. 1997; Brooks et al. 1998, for CDS and Feldman et al. 1997, for SUMER), including emission lines from many ions not accounted for in the 1.0 version of the CHIANTI database. These lines are potentially very useful for plasma diagnostic and chemical composition analysis. The SUMER instrument has also observed strong continuum radiation - a feature not accounted for in CHIANTI 1.0 .

In the present paper we present the 2.0 version of the CHIANTI database and we describe the changes and improvements made in order to better reproduce the optically thin spectrum for wavelengths greater than $50 \AA$. This has greatly increased the number of ions inserted in the database, and the changes are indicated in Tables 1 and 2 .

A detailed description of the basic structure of the CHIANTI database is given in Paper I; since no change has been made in the organization of the database, this will not be repeated here, and we refer the reader to Paper I for further details.

In the near future the attention will be focused on the spectral range $\lambda \leq 50 \AA$ in order to meet the needs of the AXAF and XMM missions, that are scheduled for launch in late-1998 and 1999, respectively. The ions necessary to 
calculate the spectrum below $50 \AA$ will be inserted in the CHIANTI database in a later version. Moreover, detailed work using the Close Coupling approximation is underway as part of the Iron Project (Hummer et al. 1993) to produce very accurate collisional and radiative data for the iron ions and for other ions as well. One of us (HEM) is a member of the Iron Project consortium and these calculations will be incorporated into CHIANTI as soon as they are available.

The CHIANTI software has been integrated into the CDS science analysis software and several useful IDL routines have been developed (they are described in the CDS software notes available online). CHIANTI has been very useful for the analysis of SOHO data (e.g. Mason et al. 1997; Landi \& Landini 1997; Del Zanna \& Bromage 1997; Brickhouse \& Esser 1997) and for studying the intensity calibration of the CDS spectrometer (Landi et al. 1997). A detailed comparison between CHIANTI and the SERTS spectrum observed in 1989 (Thomas \& Neupert 1994) has shown an overall excellent agreement between observed intensities and CHIANTI predictions (Young et al. 1998, Paper II).

\section{The beryllium isoelectronic sequence}

\subsection{Ne VII}

The new $R$-matrix calculations of Ramsbottom et al. (1994, 1995) are adopted in the CHIANTI 2.0 version. These calculations provide thermally-averaged collision strengths for transitions between the 9 lowest configurations $\left(2 \mathrm{~s}^{2}, 2 \mathrm{~s} 2 \mathrm{p}, 2 \mathrm{p}^{2}, 2 \mathrm{~s} 3 \mathrm{~s}, 2 \mathrm{~s} 3 \mathrm{p}, 2 \mathrm{~s} 3 \mathrm{~d}, 2 \mathrm{p} 3 \mathrm{~s}, 2 \mathrm{p} 3 \mathrm{p}\right.$ and $2 \mathrm{p} 3 \mathrm{~d}$ ) corresponding to 46 fine-structure energy levels. The experimental energy levels are taken from the NIST database (Martin et al. 1995). Radiative transition probabilities and theoretical energy levels come from the same source as in the CHIANTI v. 1.0 version (Zhang \& Sampson 1992; Muhlethaler \& Nussbaumer 1976), although the original Ramsbottom et al. $(1994,1995)$ theoretical energies have been used for fitting the thermallyaveraged collision strengths in CHIANTI format.

For some transitions the behaviour of the thermallyaveraged collision strengths as a function of electron temperature is quite complex and the 5-point spline interpolation is not able to reproduce with the required accuracy their dependence on $T_{\mathrm{e}}$. For this reason values of the thermally-averaged collision strengths for temperatures lower than $10^{4.7} \mathrm{~K}$ have been omitted from the database, so that the interpolation technique could reproduce adequately the behaviour of the curve. The omission of these points should not have any consequence as they are outside the temperature range in which Ne VII has significant population.

The use of the $R$-matrix thermally-averaged collision strengths instead of the Distorted Wave collision strengths has significant effects in the lower level populations and theoretical line intensities.

\section{2. $M g I X$}

A problem with the original CHIANTI model of Mg IX was that level $26\left(3 \mathrm{p} 3 \mathrm{~d}{ }^{3} \mathrm{~F}_{4}\right)$ became incorrectly metastable on account of the absence of published allowed transition probabilities to de-populate the level, although the level would clearly decay via allowed transitions to the 2s $3 \mathrm{~d}^{3} \mathrm{D}_{3}$ and $2 \mathrm{p} 3 \mathrm{p}{ }^{3} \mathrm{D}_{3}$ levels. To correct this in v. 1.0 of CHIANTI, the $g f$ values for these transitions were taken from the CHIANTI O v model (Hibbert 1980) and scaled with $\mathrm{Mg}$ IX energies to yield $A$-values for the transitions.

For a more correct approach, it was decided to use SSTRUCT (Eissner et al. 1974; see also Sect. 3 of Paper I) to calculate $A$-values for all transitions between the 46 levels of the CHIANTI model. This data is now in the CHIANTI 2.0 database. Care was taken to ensure that the SSTRUCT level ordering matched that in the CHIANTI database. The SSTRUCT model included the $3 \mathrm{~s}^{2}, 3 \mathrm{p}^{2}, 3 \mathrm{~d}^{2}, 3 \mathrm{~s} 3 \mathrm{p}, 3 \mathrm{~s} 3 \mathrm{~d}$ and $3 \mathrm{p} 3 \mathrm{~d}$ configurations in addition to the 9 configurations of the CHIANTI model already mentioned for Ne VII.

\subsection{SXIII, ArXV, CaXVII, FeXXIII, NiXXV}

The SxiII, Ar Xv, CaxviI, FexxiII and Nixxv atomic models have been extended up to 46 fine-structure energy levels, including the $n=3$ configurations. These additional configurations were already part of the 1.0 version of the database for the lighter ions. Experimental energy levels come from the NIST database (Martin et al. 1995) for all the ions, but have been complemented with other sources where necessary: Khardi et al. (1994) (S xiII, ArXv), Martin et al. (1990) (S XIII), Sugar \& Corliss (1985) (Ca XVII) and Kelly (1987) (CaXVII). Theoretical energy levels are taken from Zhang \& Sampson (1992) and from Sampson et al. (1984).

Radiative and collisional transition probabilities for transitions from the $n=3$ levels have been taken from Sampson et al. (1984). The authors provide CoulombBorn-exchange collision strengths calculated for nine values of the incident electron energy $\epsilon$ in threshold units, in the range $1 \leq \epsilon \leq 15$. Also electric dipole radiative transition probabilities for transitions from the $n=3$ levels are supplied.

\section{The boron isoelectronic sequence}

\section{1. $C a X V I$}

An important collision strength was left out of the Ca XVI files in version 1.0. This caused significant alterations in 
Table 1. Ions included in the CHIANTI database. •: Ions in the CHIANTI 1.0 version not changed in the present update. $\circ$ : Ions in the CHIANTI 1.0 version whose data have been modified/complemented in the present update. $\star$ : New entries for the CHIANTI database

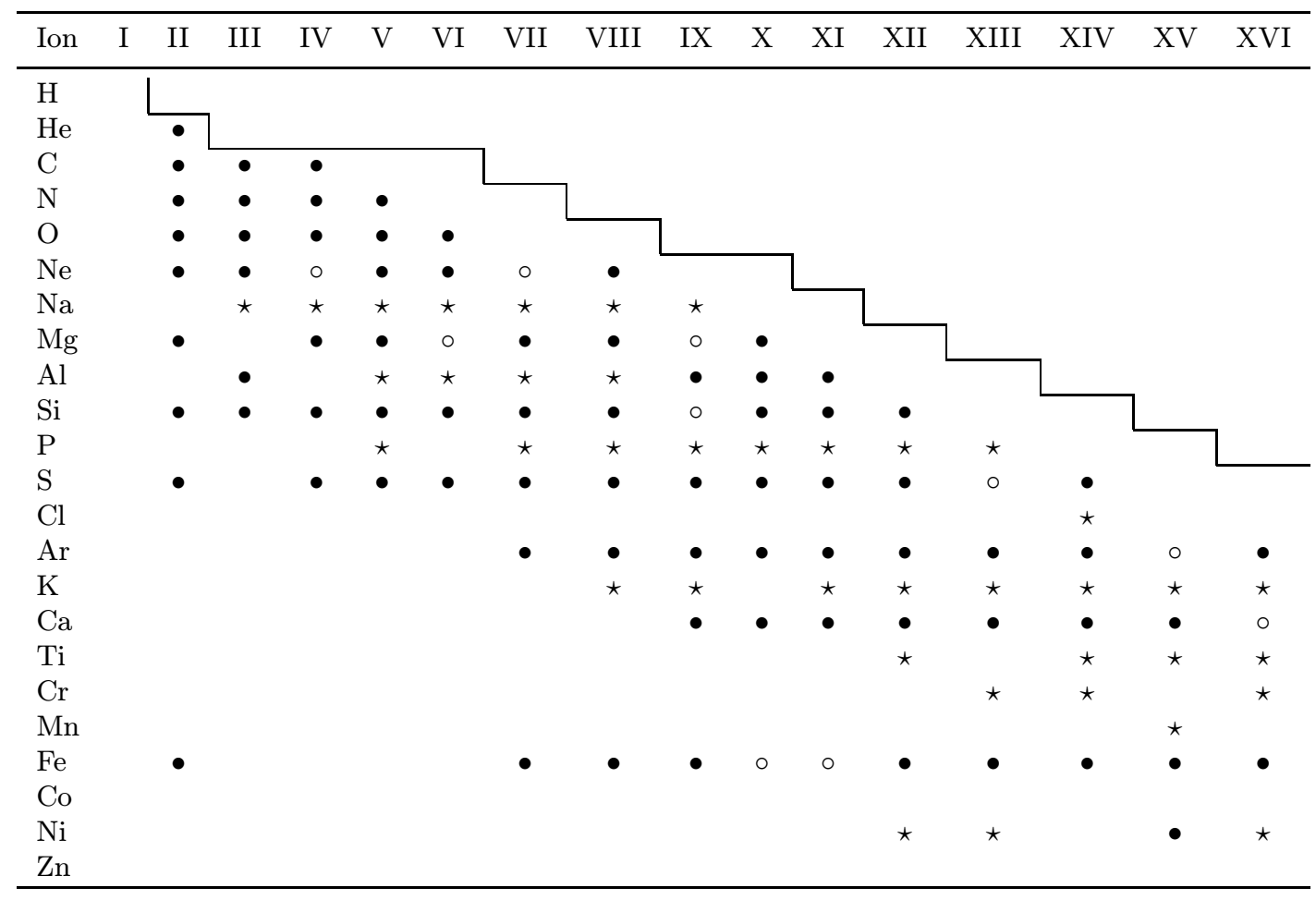

Table 2. Ions included in the CHIANTI database. •: Ions in the CHIANTI 1.0 version not changed in the present update. $\circ$ : Ions in the CHIANTI 1.0 version whose data have been modified/complemented in the present update. $\star$ : New entries for the CHIANTI database

\begin{tabular}{|c|c|c|c|c|c|c|c|c|c|c|c|c|}
\hline Ion & XVII & XVIII & XIX & $\mathrm{XX}$ & XXI & XXII & XXIII & XXIV & XXV & XXVI & XXVII & XXVIII \\
\hline $\mathrm{Ar}$ & & & & & & & & & & & & \\
\hline $\mathrm{K}$ & $\star$ & & & & & & & & & & & \\
\hline $\mathrm{Ca}$ & $\circ$ & $\bullet$ & & & & & & & & & & \\
\hline $\mathrm{Ti}$ & $\star$ & $\star$ & $\star$ & $\star$ & & & & & & & & \\
\hline $\mathrm{Cr}$ & $\star$ & $\star$ & $\star$ & $\star$ & $\star$ & $\star$ & & & & & & \\
\hline Mn & $\star$ & $\star$ & $\star$ & $\star$ & $\star$ & $\star$ & $\star$ & & & & & \\
\hline $\mathrm{Fe}$ & $\bullet$ & $\bullet$ & $\bullet$ & $\circ$ & $\bullet$ & $\bullet$ & $\circ$ & $\bullet$ & & & & \\
\hline $\mathrm{Co}$ & $\star$ & & $\star$ & & & $\star$ & $\star$ & $\star$ & $\star$ & & & \\
\hline $\mathrm{Ni}$ & • & $\bullet$ & • & • & & & $\star$ & $\bullet$ & $\bullet$ & • & & \\
\hline $\mathrm{Zn}$ & & & & $\star$ & & & & $\star$ & $\star$ & $\star$ & $\star$ & \\
\hline
\end{tabular}

the level population and line emissivity calculations for some spectral lines and has been corrected in the present version of the CHIANTI database.

\section{The carbon isoelectronic sequence}

\subsection{Al VIII and NiXXIII}

The adopted Al VIII and Ni XXIII models include the three $n=2$ configurations $\left(2 \mathrm{~s}^{2} 2 \mathrm{p}^{2}, 2 \mathrm{~s} 2 \mathrm{p}^{3}\right.$ and $\left.2 \mathrm{p}^{4}\right)$ corresponding to 20 fine-structure energy levels. Experimental energy levels are taken from the NIST database (Martin et al. 1995), with the exception of the $2 \mathrm{~s} 2 \mathrm{p}^{3}{ }^{3} \mathrm{P}$ levels coming from Edlen (1985) in the case of Al VIII. Theoretical energy levels come from Zhang \& Sampson (1996).

Radiative transition probabilities come from Zhang \& Sampson (1996) (electric dipole) and Galavis et al. (1997) for the ground levels. Zhang \& Sampson (1996) relativistic Distorted Wave collision strengths have been adopted; they provide collision strengths for all possible transitions calculated for six values of the incident electron energy. 


\subsection{SiIX}

The $R$-matrix calculations of Aggarwal (1983) have been used to provide collisional data for the ground transitions of Si IX. For all other transitions, the Distorted Wave collision strengths of Bhatia \& Doschek (1993, 1995) have been retained. The inclusion of resonances for the ground transitions have significant effects in the determination of densities from the Si IX density diagnostics.

\section{The nitrogen isoelectronic sequence}

\subsection{Ne IV}

Ramsbottom et al. (1998) have recently presented thermally-averaged collision strengths for Ne IV calculated in the $R$-matrix approximation. Values were presented over the temperature range $3.6 \leq \log T \leq 6.1$. This data replaces the Distorted Wave calculations of Bhatia (1996, unpublished) that were previously in the database.

For the same reasons explained in Sect. 2.1, thermallyaveraged collision strengths for temperatures lower than $10^{4.4} \mathrm{~K}$ have been omitted from the database.

The new model for the ion includes the 22 finestructure levels of the $2 \mathrm{~s}^{2} 2 \mathrm{p}^{3}, 2 \mathrm{~s} 2 \mathrm{p}^{4}, 2 \mathrm{p}^{5}$ and $2 \mathrm{~s}^{2} 2 \mathrm{p}^{2} 3 \mathrm{~s}$ configurations, whose experimental energies come from the NIST database and Bhatia \& Kastner (1988). Theoretical energy levels and thermally-averaged collision strengths come from the calculations of Ramsbottom et al. (1998). Radiative transition probabilities have been calculated using the SSTRUCT package including 17 configurations: $2 \mathrm{~s}^{2} 2 \mathrm{p}^{3}, 2 \mathrm{~s} 2 \mathrm{p}^{4}, 2 \mathrm{p}^{5}, 2 \mathrm{~s}^{2} 2 \mathrm{p}^{2} 3 \mathrm{l}, 2 \mathrm{~s} 2 \mathrm{p}^{3} 3 \mathrm{l}(l=s, p, d), 2 \mathrm{~s}^{2} 2 \mathrm{p}^{2} 4 \mathrm{l}$ and $2 \mathrm{~s} 2 \mathrm{p}^{3} 4 \mathrm{l}(l=s, p, d, f)$.

Comparison between Ramsbottom et al. (1998) and Bhatia (1996) has shown that differences of up to $40 \%$ arise between theoretical emissivities calculated from the two models.

\section{2. $M g V I$}

The new Distorted Wave calculations of Bhatia \& Young (1998) have been fitted and replace the data of Bhatia \& Mason (1980b) in the database. The new calculations include the configurations $2 \mathrm{~s}^{2} 2 \mathrm{p}^{3}, 2 \mathrm{~s} 2 \mathrm{p}^{4}, 2 \mathrm{p}^{5}$ and $2 \mathrm{~s}^{2} 2 \mathrm{p}^{2} 3 \mathrm{~s}$ and were computed at several values of the incoming electron energy. Radiative data are also taken from Bhatia \& Young (1998). The level energies of Edlen (1984) are used for the $2 \mathrm{~s}^{2} 2 \mathrm{p}^{3}, 2 \mathrm{~s} 2 \mathrm{p}^{4}$ and $2 \mathrm{p}^{5}$ configurations, while the $2 \mathrm{~s}^{2} 2 \mathrm{p}^{2} 3 \mathrm{~s}$ energies are from the NIST database (Martin et al. 1995).

A comparison between the Bhatia \& Young (1998) collision strengths and the $R$-Matrix thermally-averaged collision strengths calculated by Ramsbottom \& Bell (1997) did not show any significant differences in level populations and line intensities.

\subsection{Al VII and NiXXII}

The adopted atomic model for Al VII and Ni XXII includes the $2 \mathrm{~s}^{2} 2 \mathrm{p}^{3}$ and $2 \mathrm{~s} 2 \mathrm{p}^{4}$ configurations corresponding to 13 fine-structure energy levels. Experimental energy levels have been taken from the NIST database (Martin et al. 1995) for Al VII and from Edlen (1984) for Ni XXII.

Due to the lack of atomic data in the literature, collision strengths, radiative transition probabilities and energy levels have been interpolated along the isoelectronic sequence for both ions. The data used for interpolation were taken from the most abundant ions of the sequence, already included in version 1.0 of the CHIANTI database. For Ni XxII, the Zn XxIV data from Bhatia et al. (1989) have been included in the interpolation.

The interpolation program has been developed by one of the authors (EL) and allows the calculation of theoretical energy levels, radiative transition probabilities and thermally-averaged collision strengths through the interpolation of CHIANTI data for isoelectronic ions. Extensive checks have been made in order to assess the reliability of the results: the use of the program on ions already present in the CHIANTI database yielded results within $5 \%$ of the original data.

\subsection{Fe $X X$}

The 13 level Fe xx model described in Paper I has been extended to 72 levels through the inclusion of the the $2 \mathrm{~s}^{2} 2 \mathrm{p}^{2} 3 \mathrm{l}(l=s, p, d)$ configurations, corresponding to 72 fine-structure energy levels. The experimental energy levels have been taken from the NIST database (Martin et al. 1995), replacing also the values of the lower $n=2$ levels adopted in CHIANTI v. 1.0 from Bhatia \& Mason (1980a). Theoretical energy levels are from Bhatia \& Mason (1980a) ( $n=2$ configurations) and Bhatia et al. (1989) ( $n=3$ configurations).

Radiative and collisional transition probabilities for the $n=3$ transitions are taken from the single-energy Distorted Wave calculations of Bhatia et al. (1989). The availability of only one energy point for $\Omega$ did not allow to take into account the dependence of $\Omega$ on the electron energy and therefore the calculation of the thermallyaveraged collision strengths is somewhat arbitrary. For transitions with nonzero values of the oscillator strenght, the scaled collision strengths were obtain by a linear fit to the given collision strength and the high energy limit determined from the oscillator strength. For forbidden lines, the scaled collision strength was assumed to be constant with energy.

The Bhatia \& Mason (1980a) radiative and collisional transition probabilities have been maintained. 


\section{The oxygen isoelectronic sequence}

\subsection{Al VI}

The $n=2$ configurations $2 \mathrm{~s}^{2} 2 \mathrm{p}^{4}, 2 \mathrm{~s} 2 \mathrm{p}^{5}$ and $2 \mathrm{p}^{6}$ have been included in the CHIANTI database, corresponding to 10 fine-structure energy levels. The experimental energy levels come from the NIST database (Martin et al. 1995).

Radiative transition probabilities for the ground configuration are taken from Galavis et al. (1997), while allowed $A$ values come from Fawcett (1986a). Butler \& Zeippen (1994) provide thermally-averaged collision strengths for the ground level transitions over a large temperature range. These calculations were performed using the Close Coupling approximation as part of the Iron Project. There are no other sources of collisional data in the literature, and so for transitions involving the levels of the $2 \mathrm{~s} 2 \mathrm{p}^{5}$ and $2 \mathrm{p}^{6}$ configurations, the data have been interpolated from other oxygen-like ions already present in the CHIANTI database, using the program mentioned in Sect. 5.3.

\section{The fluorine isoelectronic sequence}

\section{1. $A l V$}

A model containing the three fine-structure levels of the $2 \mathrm{~s}^{2} 2 \mathrm{p}^{5}$ and $2 \mathrm{~s} 2 \mathrm{p}^{6}$ configurations has been adopted for $\mathrm{Al} \mathrm{V}$. Experimental energy levels come from the NIST database (Martin et al. 1995).

Due to the neglect of this ion in the literature, theoretical energy levels, radiative and collisional transition probabilities have been interpolated using all the other isoelectronic ions already inserted in the old version of the CHIANTI database. The program mentioned in Sect. 5.3 has been used.

\section{The aluminum isoelectronic sequence}

\subsection{Ni XVI}

The $3 \mathrm{~s}^{2} 3 \mathrm{p}, 3 \mathrm{~s} 3 \mathrm{p}^{2}, 3 \mathrm{~s}^{2} 3 \mathrm{~d}, 3 \mathrm{p}^{3}$ and $3 \mathrm{~s} 3 \mathrm{p} 3 \mathrm{~d}$ configurations are included in the CHIANTI model for NixVI, corresponding to 40 fine-structure energy levels. Experimental energy levels come from the NIST database (Martin et al. 1995), but unfortunately no entry is found for any of the 3s3p3d levels. All the data are taken from Bhatia \& Doschek (1998b), reporting theoretical energy levels, radiative transition probabilities and collision strengths for all possible transitions between the included 40 levels.

Collision strengths have been calculated for five values of the incident electron energy (10 to $50 \mathrm{Ry}$ ) using the Distorted Wave approximation. To our knowledge, this is the first large scale calculation for Ni XVI collision strengths. Close Coupling calculations are in progress at the University College of London (Storey 1998) and these will be assessed as soon as they are available.

The only NixVI comprehensive radiative transition probabilities calculations we are aware of are those by Huang (1986), showing reasonable agreement with the Bhatia \& Doschek (1998b) values.

\section{The sulphur isoelectronic sequence}

\section{1. $\mathrm{Fe} X I$}

Previously, only the strongest transitions into and out of the metastables had been fitted for this ion from Bhatia \& Doscheck (1996). The remaining transitions have now been added. This was found to change the level populations for some of the metastables and, in the worst case $\left(3 \mathrm{~s}^{2} 3 \mathrm{p}^{3} 3 \mathrm{~d}{ }^{5} \mathrm{D}_{4}\right)$, the population was lowered by $20 \%$. However, this has a negligible effect on the Fe XI allowed transitions.

\subsection{NiXIII}

The adopted atomic model for Ni XIII includes 4 different configurations $\left(3 \mathrm{~s}^{2} 3 \mathrm{p}^{4}, 3 \mathrm{~s} 3 \mathrm{p}^{5}, 3 \mathrm{~s}^{2} 3 \mathrm{p}^{3} 3 \mathrm{~d}\right.$ and $\left.3 \mathrm{p}^{6}\right)$ corresponding to 48 fine-structure energy levels. Experimental energy levels are taken from the NIST database, although for the majority of the $3 \mathrm{~s}^{2} 3 \mathrm{p}^{3} 3 \mathrm{~d}$ energy levels no value is available.

Theoretical energy levels, radiative transition probabilities and collision strengths are taken from Bhatia \& Doscheck (1998a). The authors provide Distorted Wave collision strengths for all possible transitions between the included levels; these are calculated for five values of the incident electron energy between 10 and $50 \mathrm{Ry}$. To our knowledge this is the first complete calculation for Ni XIII collision strengths.

Radiative transition probabilities have been compared with previous calculations (Fawcett 1986b; Saloman \& Kim 1989), and good agreement has been found.

\section{The chlorine isoelectronic sequence}

\subsection{Fe $X$}

As with Fe XI (see Sect. 9.1), only the strongest transitions into and out of the metastables had been fitted for Paper I. The remaining transitions have now been added. In addition, due to large discrepancies found for the allowed $3 \mathrm{~s}^{2} 3 \mathrm{p}^{5}-3 \mathrm{~s}^{2} 3 \mathrm{p}^{4} 3 \mathrm{~d}$ transitions when comparing the Bhatia \& Doschek (1995) model and the more detailed SSTRUCT model (described in Sect. 4.18 of Paper I), it was decided to scale the collision strengths for these transitions via the method of Flower \& Nussbaumer (1974).

These changes serve to lower the theoretical emissivities of the allowed transitions, while also the populations of the metastables are altered by up to $30 \%$. 


\subsection{NiXII}

The adopted atomic model for Ni XII includes three configurations $\left(3 \mathrm{~s}^{2} 3 \mathrm{p}^{5}, 3 \mathrm{~s} 3 \mathrm{p}^{6}\right.$ and $\left.3 \mathrm{~s}^{2} 3 \mathrm{p}^{4} 3 \mathrm{~d}\right)$ corresponding to 31 fine-structure energy levels. Experimental energy levels are taken from the NIST database (Martin et al. 1995).

Theoretical energy levels and radiative transition probabilities have been calculated using SSTRUCT. The following cofigurations were included: $3 \mathrm{~s}^{2} 3 \mathrm{p}^{5}$, $3 \mathrm{~s} 3 \mathrm{p}^{6}, 3 \mathrm{~s}^{2} 3 \mathrm{p}^{4} 3 \mathrm{~d}, 3 \mathrm{~s} 3 \mathrm{p}^{5} 3 \mathrm{~d}, 3 \mathrm{p}^{6} 3 \mathrm{~d}, 3 \mathrm{~s}^{2} 3 \mathrm{p}^{3} 3 \mathrm{~d}^{2}, 3 \mathrm{~s} 3 \mathrm{p}^{4} 3 \mathrm{~d}^{2}$, $3 \mathrm{p}^{5} 3 \mathrm{~d}^{2}, 3 \mathrm{~s}^{2} 3 \mathrm{p}^{2} 3 \mathrm{~d}^{3}, 3 \mathrm{~s} 3 \mathrm{p}^{3} 3 \mathrm{~d}^{3}, 3 \mathrm{p}^{4} 3 \mathrm{~d}^{3}, 3 \mathrm{~s}^{2} 3 \mathrm{p}^{4} 4 \mathrm{~s}, 3 \mathrm{~s}^{2} 3 \mathrm{p}^{4} 4 \mathrm{~d}$, $3 \mathrm{~s}^{2} 3 \mathrm{p}^{4} 4 \mathrm{f}$. The resulting $A$ values and oscillator strengths have been compared with the calculations by Fawcett (1987), showing reasonable agreement.

Ni XII thermally-averaged collision strengths have been calculated by Matthews et al. (1998) in the temperature range $10^{3.2}-10^{6.6} \mathrm{~K}$. The authors used the $R$-Matrix method. To our knowledge, these are the only largescale electron-ion collision calculations performed in the literature.

For the same reasons as described in Sect. 2.1, thermally-averaged collision strengths for temperatures lower than $10^{4.7} \mathrm{~K}$ have been omitted from the database.

\section{Continuum radiation}

CHIANTI v. 2.0 allows the calculation of continuum radiation according to the Gronenschild \& Mewe (1978) model. Here we provide only the formulas adopted for the calculation, and further details can be found in the quoted paper.

The computation includes free-free, free-bound and two-photon continuum and the results are given in units $10^{-20} \mathrm{erg} \mathrm{cm}^{3} \mathrm{~s}^{-1} \AA^{-1}$.

An IDL routine is supplied together with the database which allows one to calculate the continuum radiation for temperatures between $10^{4}$ to $10^{8} \mathrm{~K}$ and to convolve the result with a selected $D E M$ in order to calculate the continuum radiation synthetic spectrum.

The approximations used in the adopted model are accurate to better than $15 \%$.

It is important to note that the Gronenschild \& Mewe (1978) model for continuum emission adopted in the present version of CHIANTI has been successively improved by Mewe \& Kaastra (1994). The improvements include:

- Free-free radiation: the approximation to the correction for relativistic effects based on the calculations of Kylafis \& Lamb (1982) and the interpolation of the results given by Carson (1988) which replace the older Karzas \& Latter (1961) calculations;

- Free-bound radiation: the full energy dependence of the Gaunt factor has been taken into account;

- Two-photon emission: The accuracy of the calculation has been improved to better than $\simeq 1 \%$.
These improvements will be included in a future release of the CHIANTI database.

\subsection{Free-free radiation}

The free-free continuum energy emitted by a plasma of a given chemical composition per unit time, volume and wavelength band in the hydrogenic approximation is given by

$$
\begin{array}{r}
\frac{\mathrm{d} P_{\mathrm{ff}}(\lambda, T)}{\mathrm{d} \lambda}=C_{\mathrm{ff}} \frac{N_{\mathrm{e}} \mathrm{e}^{-\frac{h c}{\lambda k T}}}{\lambda^{2} T^{1 / 2}} \times \\
\times \Sigma_{Z, z} \frac{N\left(Z^{+z}\right)}{N(Z)} \frac{N(Z)}{N(H)} N(H) z^{2} g_{\mathrm{ff}}(z, \lambda, T)
\end{array}
$$

$\lambda$ is the radiation wavelength (in $\AA$ ), $N_{\mathrm{e}}$ is the electron density (in $\left.\mathrm{cm}^{-3}\right), \frac{N\left(Z^{+z}\right)}{N(Z)}$ is the ion $Z^{+z}$ abundance relative to the total abundance of the element, $\frac{N(Z)}{N(H)}$ is the element $Z$ abundance relative to hydrogen and $C_{\mathrm{ff}}=2.05110^{-19} \cdot g_{\mathrm{ff}}(z, \lambda, T)$ is the temperature-averaged free-free hydrogenic Gaunt factor, evaluated in the same way as Gronenschild \& Mewe (1978).

In the present version only $\mathrm{HI}$, He I and He II are included in the computation.

\subsection{Free-bound radiation}

The free-bound continuum energy emitted by a plasma of given chemical composition per unit time, volume and wavelength band is given by

$$
\begin{array}{r}
\frac{\mathrm{d} P_{\mathrm{fb}}(\lambda, T)}{\mathrm{d} \lambda}=\frac{C_{\mathrm{ff}}}{\lambda^{2} T^{1 / 2}}\left(\frac{I_{\mathrm{H}}}{k T}\right) N_{\mathrm{e}} \mathrm{e}^{-\frac{h c}{\lambda k T}} \times \\
\times \Sigma_{Z, z, n} g_{f b}\left(\frac{\eta_{Z, z, n}}{n}\right)\left(\frac{I_{Z, z-1, n}}{k t}\right)^{2} \times \\
\times \frac{N\left(Z^{+z}\right)}{N(Z)} \frac{N(Z)}{N(H)} N(H) \mathrm{e}^{\frac{I_{Z, z-1, n}}{k t}}
\end{array}
$$

with

$\frac{I_{Z, z-1, n}}{k t} \leq \frac{h c}{\lambda k T}$

where $I_{Z, z-1, n}=I_{H} \frac{z^{2}}{n^{2}}$ is the ionization energy of the $n$ state of the recombined ions, $\eta_{Z, z, n}$ is the number of position of the $n^{\text {th }}$ shell of the recombining ion free to be occupied by the captured electron. The free-bound Gaunt factor $g_{\mathrm{fb}}$ is equal to 1 following Karzas \& Latter (1961) and taking into account contribution of recombination to excited levels also.

The elements to be included in the computation may be selected via an option on the chemical abundance. 


\subsection{Two-photon radiation}

The two-photon continuum energy emitted by a plasma composed by hydrogen and helium-like ions per unit time, volume and wavelength band is given by

$$
\begin{array}{r}
\frac{\mathrm{d} P_{2 \mathrm{ph}}(\lambda, T)}{\mathrm{d} \lambda}=\frac{2.72710^{-15}}{\lambda^{3} T^{-1 / 2}} N_{\mathrm{e}} \times \\
\times \Sigma_{Z, z} \frac{N\left(Z^{+z}\right)}{N(Z)} \frac{N(Z)}{N(H)} N(H) \times \\
\times f_{Z, z} g_{Z, z} \lambda_{Z, z}{ }^{2} \mathrm{e}^{-\frac{h c}{\lambda Z, z^{k T}}} \Phi(y) \quad\left(\lambda \geq \lambda_{Z, z}\right)
\end{array}
$$

where

$$
\Phi(y)=2.623 \cdot \sqrt{\cos [\pi(y-1 / 2)]}
$$

and $f_{Z, z}$ and $\lambda_{Z, z}$ are the absorption oscillator strength and the wavelength of the $1 \mathrm{~s}-2 \mathrm{~s}$ transition, $g_{Z, z}$ is the averaged Gaunt factor and $y=\frac{\lambda_{Z, z}}{\lambda}$.

$I_{Z, z, n}$ and $\eta(Z, z, n)$ for the free-bound continuum and $f_{Z, z}, \lambda_{Z, z}$ and $g_{Z, z}$ for the two-photon continuum are supplied in a proper file. Reduction of the two-photon continuum emission by depopulation of the $2 \mathrm{~s}$ level via collisional de-excitation is taken into account. For this purpose the electron density selection is allowed.

\section{The minor ions dataset}

The 2.0 version of the CHIANTI database includes energy levels, radiative transition probabilities and collision strengths for a large number of ions belonging to the socalled Minor Elements: Na, P, Cl, K, Ti, Cr, Mn, Co, Zn. Lines from these ions have often been observed in the past both in solar and stellar spectra, and they can be useful for plasma diagnostics.

In the recent past a careful review of the existing Minor Ions data in the literature has been carried out by Landi \& Landini (1998), including data for the Minor Elements belonging to the lithium, beryllium, boron, carbon, nitrogen, oxygen, fluorine, sodium and magnesium isoelectronic sequences.

In the 2.0 version of the CHIANTI database we include the Minor Ions database resulting from Landi \& Landini (1998), where details on the atomic data used can be found. The list of the ions included in the CHIANTI database is given in Tables 1 and 2 .

In order to calculate line emissivities with CHIANTI, ionization equilibrium populations are necessary, and we have adopted the Landini \& Monsignori Fossi (1991) results. A file containing the Landini \& Monsignori Fossi (1991) ionization equilibrium calculations is distributed with version 2.0 of the CHIANTI database.

A new calculation of ionization balance for all elements from helium to nickel has been performed by Mazzotta et al. (1998). These dataset will be inserted into CHIANTI as soon as it will be available.

\section{Conclusions}

The present paper describes version 2.0 of the CHIANTI database, including data for a large number of ions not included in the present version of the database, more recent transition probabilities for ions already included in the database, and a full set of data and IDL routines to calculate free-free, free-bound and two-photon continuum radiation.

The present version of the database allows a better reproduction of the optically thin spectrum for wavelengths greater than $50 \AA$ and is able to account for most of the lines observed by the most advanced spectrometers.

In principle, there is no upper limit to the CHIANTI wavelength coverage, but beyond $1100 \AA$ lines from neutral species begin to dominate the spectrum and neutrals are not presently included in the CHIANTI database. The database is still incomplete below $50 \AA$ mostly because of the omission of the hydrogen and helium isoelectronic sequences. In view of the forthcoming missions such as AXAS $(1-140 \AA)$, XMM $(5-35 \AA)$ and ASTRO-E (1 $30 \AA)$ it is important to have also a database covering wavelengths shorter than $50 \AA$ : a new release of the CHIANTI database will extend the covered spectral range to these wavelengths. It is to be noted that recently the atomic data for the H-like sequence have been assessed and critically evaluated by the Utrecht group (Kaastra 1998) as part of a major update of the MEKAL code; this careful and extensive work will be a starting point for the future extension of the CHIANTI database. Also, the Mewe \& Kaastra (1994) improvements to the continuum model will be included in the next release of the CHIANTI database.

The CHIANTI database and the corresponding set of IDL programs are freely available from the following web sites:

http://wwwsolar.nrl.navy.mil/chianti.html http://www.arcetri.astro.it/science/chianti/chianti.html http://www.damtp.cam.ac.uk/user/e2e/chianti/chianti.html

A README file explains the procedures for copying the Unix compressed tar files to a user's computer. The IDL routines assume a Unix file structure but they could be adapted to other operating systems.

Acknowledgements. The CHIANTI consortium also includes B.J.I. Bromage and G. Del Zanna (University of Central Lancashire) and C.D. Pike (Rutherford Appleton Labortaory) who have been involved in the software development and applications to the analysis of CDS data. The financial support of the Italian Space Agency (ASI contract 1995-RS 120) and the Italian Ministry of University and Technological Development (MURST contract 40\% 1996) are aknowledged by EL and ML. HEM and PRY acknowledge the financial support of PPARC. A NATO grant has enabled the CHIANTI consortium to meet 
and for individuals to visit other institutes. We are grateful to Dr. R. Mewe for useful comments on the original manuscript.

\section{References}

Aggarwal K.M., 1983, J. Phys. B 16, L59

Bhatia A.K., Mason H.E., 1980a, A\&A 83, 380

Bhatia A.K., Mason H.E., 1980b, MNRAS 190, 925

Bhatia A.K., Kastner S.O., 1988, ApJ 332, 1063

Bhatia A.K., Seely J.F., Feldman U., 1989, ADNDT 43, 99

Bhatia A.K., Doschek G.A., 1993, ADNDT 55, 281

Bhatia A.K., Doschek G.A., 1995, ADNDT 60, 145

Bhatia A.K., Doschek G.A., 1996, ADNDT 64, 183

Bhatia A.K., Doschek G.A., 1998a, ADNDT (in press)

Bhatia A.K., Doschek G.A., 1998b, ADNDT (in press)

Bhatia A.K., Young P.R., 1998, ADNDT 68, 219

Brickhouse N.S., Esser R., 1997, ApJ 479, 470

Brooks D.H., Fischbacher G.A., Fludra A., Harrison R.A., Innes D., Landi E., Landini M., Lang J., Lanzafame A.C., Loch S.D., McWhirter R.W.P., Summers H.P., Thompson W.T., 1998, The NIS quiet Sun spectrum, A\&A (in press)

Butler K., Zeippen C.J., 1994, A\&AS 108, 1

Carson T.R., 1988, A\&A 189, 319

Del Zanna G., Bromage B.J.I., 1997, Proceedings of the Fifth SOHO Workshop, Oslo, Norway, 17-20 June 1997, ESA SP-404, p. 323

Dere K.P., Landi E., Mason H.E., Monsignori Fossi B.C., Young P.R., 1997, A\&AS 125, 149 (Paper I)

Edlen B., 1984, Phys. Scr. 30, 135

Edlen B., 1985, Phys. Scr. 31, 345

Eissner W., Jones M., Nussbaumer H., 1974, Comp. Phys. Comm. 8, 270

Fawcett B.C., 1986a, ADNDT 34, 215

Fawcett B.C., 1986b, ADNDT 35, 185

Fawcett B.C., 1987, ADNDT 36, 151

Feldman U., Behring W.E., Curdt W., Schuehle U., Wilhelm K., Lemaire P., Moran T.M., 1997, ApJSS 113, 195

Flower D.R., Nussbaumer H., 1974, A\&A 31, 353

Galavis M.E., Mendoza C., Zeippen C.J., 1997, A\&AS 123, 159

Gronenschild E.H.B.M., Mewe R., 1978, A\&AS 32, 283

Harrison R.A., et al., 1995, Sol. Phys. 162, 233

Harrison R.A., et al., 1997, Sol. Phys. 170, 123

Hibbert A., 1980, J. Phys. B. 13, 1721

Huang K.N., 1986, ADNDT 34, 1

Hummer D.G., Berrington K.A., Eissner W., Pradhan A.K., Saraph H.E., Tully J.A., 1993, A\&A 279, 298

Kaastra J.S., 1998, in The hot universe, Koyama K., Kimamoto
S., Itoh M. (eds.). Proc. IAU Symp. No. 188, Kyoto, Japan. Kluwer, Dordrecht-Holland, p. 43

Karzas W.J., Latter R., 1961, ApJ 6, 167

Kelly R.L., 1987, J. Phys. Chem. Ref. Data 16, Suppl. No. 1

Khardi S., Buchet-Poulizac M.C., Buchet J.P., Carré M., Denis A., Désesquelles J., Livingston A.E., Martin S., Ouerdane Y., 1994, Phys. Scr. 49, 571

Kylafis N.D., Lamb D.Q., 1982, ApJSS 48, 239

Landi E., Landini M., Pike C.D., Mason H.E., 1997, Sol. Phys. 175,553

Landi E., Landini M., 1997, A\&A 327, 1230

Landi E., Landini M., 1998, A\&AS (in press)

Landini M., Monsignori Fossi B.C., 1991, A\&AS 91, 183

Martin W.C., Zalubas R., Musgrove A., 1990, J. Phys. Chem. Ref. Data 19, 821

Martin W.C., Sugar J., Musgrove A., Dalton G.R., 1995, NIST Database for Atomic Spectroscopy, Version 1.0, NIST Standard Reference Database 61

Mason H.E., Young P.R., Pike C.D., Harrison R.A., Fludra A., Bromage B.J.I., Del Zanna G., 1997, Sol. Phys. 170, 143

Matthews A., Ramsbottom C.A., Bell K.L., Keenan F.P., 1998, ApJ 492, 415

Mazzotta P., Mazzitelli G., Colafrancesco S., Vittorio N., 1998, A\&AS (in press)

Mewe R., Kaastra J.S., 1994, "Continuum Radiation Processes", SRON-Utrecht report SRON/SPEX/TRPB03 (obtainable from http://www.sron.nl/ via project HEA and SPEX)

Muhlethaler H.P., Nussbaumer H., 1976, A\&A 48, 109

Ramsbottom C.A., Berrington K.A., Bell K.L., 1994, J. Phys. B 27, L811

Ramsbottom C.A., Berrington K.A., Bell K.L., 1995, ADNDT 61, 105

Ramsbottom C.A., Bell K.L., 1997, A\&AS 125, 543

Ramsbottom C.A., Bell K.L., Keenan F.P., 1998, MNRAS 293, 233

Saloman E.B., Kim Y.K., ADNDT 41, 339

Sampson D.H., Goett S.J., Clark R.E.H., 1984, ADNDT 30, 125

Storey P.J., 1998 (private communication)

Sugar J., Corliss C., 1985, J. Phys. Chem. Ref. Data. 14, Suppl. No. 2

Thomas R.J., Neupert W.M., 1994, ApJSS 91, 461

Wilhelm K., et al., 1995, Sol. Phys. 162, 189

Young P.R., Landi E., Thomas R.J., 1998, A\&A 329, 291 (Paper II)

Zhang H.L., Sampson D.H., 1992, ADNDT 52, 143

Zhang H.L., Sampson D.H., 1996, ADNDT 63, 275 